\title{
Theory of light diffusion in disordered media with linear absorption or gain
}

\author{
A. Lubatsch and J. Kroha \\ Physikalisches Institut, Universität Bonn, 53115 Bonn, Germany
}

K. Busch

Institut für Theorie der Kondensierten Materie, Universität Karlsruhe, 76128 Karlsruhe, Germany, and Department of Physics and College of Optics \& Photonics: CREOL \& FPCE, University of Central Florida, Orlando, FL 32816

\begin{abstract}
We present a detailed, microscopic transport theory for light in strongly scattering disordered systems whose constituent materials exhibit linear absorption or gain. Starting from Maxwell's equations, we derive general expressions for transport quantities such as energy transport velocity, transport mean free path, diffusion coefficient, and absorption/gain length. The approach is based on a fully vectorial treatment of the generalized kinetic equation and utilizes an exact Ward identity (WI). While for loss- and gainless media the WI reflects local energy conservation, the effects of absorption or coherent gain are implemented exactly by novel, additional terms in the WI. As a result of resonant (Mie) scattering from the individual scatterers, all transport quantities acquire strong, frequency-dependent renormalizations, which are, in addition, characteristically modified by absorption or gain. We illustrate the influence of various experimentally accessible parameters on these quanitities for dilute systems. The transport theory presented here may set the stage for a theory of Random Lasing in three-dimensional disordered media.
\end{abstract}

PACS numbers:

\section{INTRODUCTION}

Despite its long and venerable history, light propagation in disordered media continues to be a fascinating and intensely studied topic. In particular, the discovery of the coherent back scattering peak ${ }^{1.2 .3}$ has triggered an intensive search for Anderson localization 4 of light. Clearly, the unambiguous demonstration of Anderson localization of electromagnetic radiation in an appropriate disordered dielectric medium in which dephasing and interaction effects can be neglected, would mark a major triumph of our current understanding of wave propagation.

In fact, early works reported anomalously low values of the diffusion constant in strongly scattering suspensions ${ }^{5.6}$. However, it was soon realized ${ }^{5.6 .7}$ that these low values of the diffusion constant are associated with the resonant (Mie) scattering of individual scatterers. This leads to a frequency-dependent dwell-time that has to be added to the time-of-flight between succesive scattering events. As a result, the energy transport velocity acquires a corresponding renormalization, while the transport mean free path remains essentially unchanged ${ }^{7.8}$. Similarly, an analysis of the coherent back scattering peak together with the dependence of the transmission through disordered semiconductor powders on the sample size, suggested a scaling behavior ${ }^{9}$ consistent with the onset of Anderson localization ${ }^{10}$. However, a reexamination of these data ignited a heated debate as to how to discriminate Anderson localization from absorption effects 11.12 . Subsequent experiments on similarly strongly scattering semiconductor powders ${ }^{13}$ did not produce evidence of Anderson localization and the experimental data could be well explained using a recently developed effective medium theory that incorpo- rates the resonant scattering effects alluded to above ${ }^{14.15}$. This unsatisfactory state of affairs has generated renewed interest in determining novel and unambiguous pathways to Anderson localization of light.

One class of highly interesting systems for multiple scattering of light are disordered dielectric media whose constituent materials exhibit one or more forms of optical anisotropies. Moreover, most optical anisotropies exhibit a certain degree of tunability through external control parameters, thus creating the possibility of a tunable disorder. For instance, Faraday-activty in multiple scattering systems breaks the time reversal symmetry of Maxwell's equations, leading to profound modifications of the coherent back scattering peak ${ }^{16,17,18}$ and in transport the optical analogue of the Hall-effect has been observed $^{19}$. Similarly, disordered nematic liquid crystals exhibit anisotropic coherent back scattering 20 and anisotropic light diffusion ${ }^{21,22}$. However, the strength of multiple scattering in bulk nematic liquid crystals is determined by the fluctuations of the nematic director field and the difference between the liquid crystal's ordinary and extraordinary index of refraction. For an observation of Anderson localization of light in such systems, it will become necessary to enhance the multiple scattering effects, for instance, by infiltrating the nematic liquid crystal into the void regions of strongly scattering photonic crystals 23 .

Equally intriguing is to combine the effects of multiple scattering of light with optical gain and to investigate how the two phenomena mutually influence each other. From the optical gain point of view, diffusing light will spend more time interacting with active material in a characteristic volume than ballistically propagating light. If this interaction time exceeds the (sponta- 
neous) decay time of the active material, avalanche-like intensity bursts, induced by incoherent feedback, could occur. In fact, early theoretical work ${ }^{24}$ suggested this very possibility and has recently been observed ${ }^{25}$. From a wave propagation point of view, optical gain increases the relative weight of long trajectories in the sample and, therefore, will modify a number of wave interference effects such as coherent back scattering ${ }^{26}$ and possibly Anderson localization. Very recent experiments point to the possibility that such long trajectories provide a feedback mechanism which leads to modes with narrow laser-like emission lines that extend across the entire sample 27 . However, the coherence properties of the emitted light still need to be analyzed in order to establish that laser action is indeed taking place in these systems. True laser action from localized regions in disordered dielectric samples with optical gain, termed Random Lasing, has been observed 28.29 .30 earlier, where measurements of the photon statistics of the emitted light ${ }^{31}$ have unambiguously demonstrated a coherent laser feedback mechanism. It has been discussed in the form of varying degrees of coupling between so-called quasi-states ${ }^{32.33}$. For a recent review of multiple scattering in amplifying media, we refer the reader to Ref ${ }^{34}$. Random Lasing has potential applications ranging from micro-lasers and optical fingerprint markers ${ }^{35}$ to the detection of cancerous tissue $e^{36}$. In addition, we want to note that recently, electrically pumped Random Laser action has been achieved in Nddoped powders ${ }^{37.38}$, thus creating potential applications of these systems in omnidirectional lighting devices and displays.

Despite these exciting developments, a convincing connection between Random Lasing and Anderson localization of light has not been demonstrated as of yet. This may be attributed to the fact, that to date the theory of random amplifying media either employs purely numerical methods in one spatial dimension ${ }^{32}$ or treats the multiple scattering part within certain approximation schemes that cannot account for the interference effects that lead to Anderson localization. These schemes include modeling the electromagnetic wave propagation through diffusion equations for the intensity ${ }^{39,40}$ or within the so-called ladder-approximation of the BetheSalpeter equation $\stackrel{41}{ }$. As a result, it is unclear, whether Anderson localization of light is a necessary condition for true Random Lasing (coherent feedback) nor whether the modified coherence properties in the lasing state have, in turn, an influence on the transition from the diffusion regime to the Anderson localized regime itself. In fact, the very concept of describing the Anderson localization transition in terms of a vanishing diffusion coefficient as an order parameter, familiar from systems with energy or particle number conservation, becomes questionable in dissipative or active media, where another channel for change of the energy density exists besides diffusion.

In the present paper, we report our progress towards answering these questions. We develop a fully vectorial transport theory for multiple scattering of light in ran- dom media whose constituent materials are isotropic and exhibit linear absorption or gain. In Section II, we derive the tensorial kinetic equation for the intensity correlation function of electromagnetic radiation. The conservation of energy in media without loss or gain is incorporated in a field theoretical way by means of an exact Ward identity (WI), the effects of loss, gain as well as frequency dependence of the material parameters being represented by additional terms. The proof of this generalized WI is presented in Section III. Subsequently, we solve the kinetic equation in the hydrodynamic limit by formulating, with the help of the WI, the continuity equation in Section IV and Fick's law in Section V, respectively. These equations relate the energy density correlation tensor and the energy current correlation tensor to each other. They allow to identify, in the hydrodynamic limit, exact expressions for quantities like the energy transport velocity, the transport mean free path and the absorption/gain length in terms of the irreducible single-photon self-energy and the two-photon irreducible vertex. We recover the well-known Mie scattering renormalization term to the transport velocity ${ }^{7.8}$, albeit in vectorial form, along with additional, characteristic renormalizations originating from absorption or gain. Section VI features numerical results for the energy velocity in dilute systems of spherical scatterers, where all quantities can be evaluated within the independent scattering approximation. The Mie resonance dips in the energy transport velocity acquire a characteristic, absorption-induced broadening and gain-induced narrowing, which may be interpreted as due to the reduction (enhancement) of higher-order scattering contributions by absorption (gain). In contrast to previous approaches, our theory can be extended to systematically include wave interference effects ("Cooperon" contributions) and, thus, to address the Anderson localization transition by virtue of a self-consistent extension ${ }^{8.43 .44 .45}$ together with a replacement of the linear absorption/gain through a coupling to the master equation of the active medium ${ }^{41}$.

\section{BASIC THEORY OF ELECTROMAGNETIC WAVE TRANSPORT}

We consider the propagation of light in a system of randomly positioned scatterers with isotropic dielectric constant $\epsilon_{s}$ immersed in a host medium with isotropic dielectric constant $\epsilon_{h}$. In addition, we allow for the possibility of having absorption or amplification in both the scatterer material and the host medium by ascribing an imaginary part to the respective dielectric constants. By virtue of the Kramers-Kronig relations between real and imaginary parts of the dielectric constant, we are required to consider materials with complex frequency dependent dielectric constant $\epsilon_{s} \equiv \epsilon_{s}(\omega)$ and $\epsilon_{h} \equiv \epsilon_{h}(\omega)$. The resulting dielectric constant

$$
\epsilon(\vec{r}, \omega)=\epsilon_{h}+\left(\epsilon_{s}-\epsilon_{h}\right) V(\vec{r}),
$$


describes the arrangement of scatterers through the function $V(\vec{r})=\sum_{\vec{R}} S_{\vec{R}}(\vec{r}-\vec{R})$ which consists of a set of localized shape functions $S_{\vec{R}}(\vec{r})$ of the invididual scatterers at random locations $\vec{R}$. The corresponding wave equation for a time harmonic wave with frequency $\omega$ and amplitude $E_{\omega}(\vec{r})$ reads

$$
\vec{\nabla} \times \vec{\nabla} \times \vec{E}_{\omega}(\vec{r})-\frac{\omega^{2}}{c^{2}} \epsilon(\vec{r}, \omega) \vec{E}_{\omega}(\vec{r})=\omega \vec{J}_{\omega}(\vec{r}) .
$$

Here, $c$ denotes the vacuum speed of light, and $\vec{J}_{\omega}(\vec{r})=$ $\left(4 \pi i / c^{2}\right) \vec{j}_{\omega}(\vec{r})$ represents an excitation of the wave field through an external current source $\vec{j}$. The dielectric function $\epsilon(\vec{r}, \omega)$ carries a positive or negative imaginary part for absorbing or coherently amplifying media, respectively.

For a specific realization $\epsilon(\vec{r}, \omega)$ of disorder, the formal solution to Eq. (1) is given in terms of the Green's tensor (of 2nd rank in the spatial vector components) $\boldsymbol{G}(\vec{r}, \vec{r}, \omega)$ as

$$
\vec{E}_{\omega}(\vec{r})=\int d^{3} r^{\prime} \boldsymbol{G}\left(\vec{r}, \vec{r}^{\prime}, \omega\right) \vec{J}_{\omega}\left(\vec{r}^{\prime}\right)
$$

For the analytical developments as well as for the comparison with experiments it is advantageous to calculate disorder-averaged quantities. Since these are translationally invariant, the transport theory must be formulated for correlation functions rather than single-particle properties. Denoting the average over disorder realizations by $\langle\ldots\rangle$, and changing to a Fourier representation of averaged quantities, the disorder averaged Green's tensor $\boldsymbol{G}^{\omega}\left(\vec{r}-\vec{r}^{\prime}\right) \equiv\left\langle\boldsymbol{G}\left(\vec{r}, \vec{r}^{\prime}, \omega\right)\right\rangle$ and its Fourier transform $\boldsymbol{G}_{\vec{k}}^{\omega}$ may be expressed in terms of the free Green's tensor $\boldsymbol{G}_{0}(\vec{k}, \omega)$ in the background medium,

$$
\boldsymbol{G}_{0}(\vec{k}, \omega)=\left(\epsilon_{h}(\omega)\left(\omega^{2} / c^{2}\right)-|\vec{k}|^{2}\right)^{-1}\left(\mathbb{1}-\hat{k} \hat{k}^{T}\right),
$$

and the self-energy tensor $\boldsymbol{\Sigma}_{\vec{k}}^{\omega}$, which represents the effects of scattering by the random perturbation or "scattering potential", $\left(\omega^{2} / c^{2}\right)\left(\epsilon_{s}(\omega)-\epsilon_{h}(\omega)\right) V(\vec{r})$, as

$$
\boldsymbol{G}_{\vec{k}}^{\omega}=\left(\boldsymbol{G}_{0}^{-1}(\vec{k}, \omega)-\boldsymbol{\Sigma}_{\vec{k}}^{\omega}\right)^{-1} .
$$

In the above expressions, we have introduced the threedimensional unit tensor $\mathbb{1}$ and the dyadic product $\hat{k} \hat{k}^{T}$ of unit vectors $\hat{k}$ in the direction of $\vec{k}$. Throughout this paper the propagators $\boldsymbol{G}$ are understood as the retarded ones and complex conjugated propagators $\boldsymbol{G}^{*}$ as the advanced ones. For practical calculations, the selfenergy tensor $\Sigma_{\vec{k}}^{\omega}$ has to be evaluated within consistent approximations such as the independent scatterer approximation (see section VI) or the Coherent Potential Approximation ${ }^{45.46}$.

The disorder-averaged field correlation tensor is

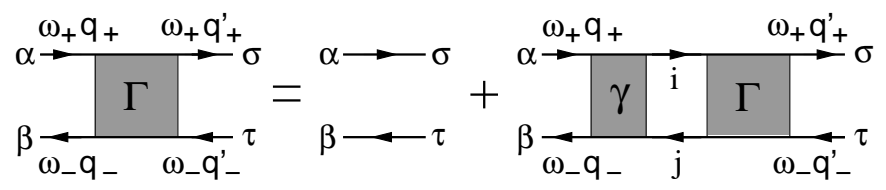

FIG. 1: Diagrammatic representation of the Bethe - Salpeter Equation, Eq. (9), indicating the tensorial structure as well as the full index notation.

definied as

$$
\begin{aligned}
\boldsymbol{I}\left(\vec{r}_{1}, \vec{r}_{2} ; \omega_{1}, \omega_{2}\right)= & \left.\left\langle\vec{E}_{\omega_{1}}\left(\vec{r}_{1}\right) \vec{E}_{\omega_{2}}^{*, T}\left(\vec{r}_{2}\right)\right]\right\rangle \\
= & \int d^{3} r_{3} \int d^{3} r_{4} \boldsymbol{\Gamma}_{\omega_{1}, \omega_{2}}\left(\vec{r}_{1}, \vec{r}_{2}, \vec{r}_{3}, \vec{r}_{4}\right) \\
& \times \boldsymbol{S}_{\omega_{1}, \omega_{2}}\left(\vec{r}_{3}, \vec{r}_{4}\right),
\end{aligned}
$$

where the system's (averaged) correlation tensor (of fourth rank) $\boldsymbol{\Gamma}_{\omega_{1}, \omega_{2}}\left(\vec{r}_{1}, \vec{r}_{2}, \vec{r}_{3}, \vec{r}_{4}\right)$ is independent of the source correlation tensor $\boldsymbol{S}_{\omega_{1}, \omega_{2}}\left(\vec{r}_{3}, \vec{r}_{4}\right)=$ $\vec{J}_{\omega_{1}}\left(\vec{r}_{3}\right) \vec{J}_{\omega_{1}}^{*} T\left(\vec{r}_{4}\right)$ and is given in terms of the disorder averaged tensor product of (unaveraged) Green's tensors according to

$$
\begin{aligned}
& \Gamma_{\omega_{1}, \omega_{2}}\left(\vec{r}_{1}, \vec{r}_{2}, \vec{r}_{3}, \vec{r}_{4}\right)= \\
&\left\langle\boldsymbol{G}\left(\vec{r}_{1}, \vec{r}_{3}, \omega_{1}\right) \otimes \boldsymbol{G}^{*}\left(\vec{r}_{2}, \vec{r}_{4}, \omega_{2}\right)\right\rangle,
\end{aligned}
$$

where $(. \otimes$.$) denotes the tensor product of two 2$ nd rank tensors operating in the space of retarded and advanced propagators, respectively. Similar to the disorder averaged Green's tensor $G_{\vec{k}}^{\omega}$, we introduce the spatial Fourier transform $\Gamma_{\vec{q} \vec{q}^{\prime}}^{\omega}(\vec{Q}, \Omega)$ of the correlation tensor

$$
\boldsymbol{\Gamma}_{\vec{q} \vec{q}^{\prime}}^{\omega}(\vec{Q}, \Omega)=\left\langle\boldsymbol{G}_{\omega_{+}}\left(\vec{q}_{+}, \vec{q}_{+}^{\prime}\right) \otimes \boldsymbol{G}_{\omega_{-}}^{*}\left(\vec{q}_{-}, \vec{q}_{-}^{\prime}\right)\right\rangle,
$$

where the transition to center of mass and relative frequencies, $\Omega, \omega$, and momenta, $\vec{Q}, \vec{q}$, respectively, with $\omega_{1,2}=\omega \pm \Omega / 2 \equiv \omega_{ \pm}, \vec{q}_{1,2}=\vec{q} \pm \vec{Q} / 2 \equiv \vec{q}_{ \pm}$and $\overrightarrow{q_{3,4}}=\vec{q}^{\prime} \pm \vec{Q} / 2 \equiv \vec{q}_{ \pm}$, facilitates an investigation of the correlation tensor's long-time $(\Omega \rightarrow 0)$ and long-distance $(|\vec{q}| \rightarrow 0)$ behavior. $\Omega, \vec{Q}$ are associated with the time and position dependence of the electromagnetic energy density in the system, while $\omega$ represents the frequency of light.

$\Gamma_{\vec{q} \vec{q}^{\prime}}^{\omega}(\vec{Q}, \Omega)$ can be expressed in terms of the irreducible vertex tensor $\boldsymbol{\gamma}_{\vec{q} \vec{q}^{\prime}}^{\omega}(\vec{Q}, \Omega)$, the two-photon analogue of the self-energy tensor, via the Bethe-Salpeter equation

$$
\begin{aligned}
\Gamma_{\vec{q} \vec{q}^{\prime}}^{\omega}(\vec{Q}, \Omega)= & \boldsymbol{G}_{\overrightarrow{q_{+}}}^{\omega_{+}} \otimes\left(\boldsymbol{G}_{\vec{q}-}^{\omega_{-}}\right)^{*}\left[(2 \pi)^{3} \delta\left(\vec{q}-\vec{q}^{\prime}\right) \mathbb{1} \otimes \mathbb{1}\right. \\
& \left.+\int \frac{d^{3} q^{\prime \prime}}{(2 \pi)^{3}} \boldsymbol{\gamma}_{\vec{q} \vec{q}^{\prime \prime}}^{\omega}(\vec{Q}, \Omega) \Gamma_{\vec{q}^{\prime \prime} \vec{q}^{\prime}}^{\omega}(\vec{Q}, \Omega)\right] .
\end{aligned}
$$

In Eq. (9), both the irreducible vertex tensor $\gamma_{\vec{q} \vec{q}^{\prime}}^{\omega}(\vec{Q}, \Omega)$ and the correlation tensor $\Gamma_{\vec{q} \vec{q}^{\prime}}^{\omega}(\vec{Q}, \Omega)$ are tensors of fourth rank which operate both in retarded and advanced space 
(see Eq. (8)). The notation $\gamma_{\vec{q} \vec{q}^{\prime}}^{\omega}(\vec{Q}, \Omega) \Gamma_{\vec{q} \vec{q}^{\prime}}^{\omega}(\vec{Q}, \Omega)$ in Eq. (9) implies contraction of both, retarded and advanced, intermediate indices,

$$
\begin{aligned}
& {\left[\boldsymbol{\gamma}_{\vec{q} \vec{q}^{\prime \prime}}^{\omega}(\vec{Q}, \Omega) \Gamma_{\vec{q}^{\prime \prime} \vec{q}^{\prime}}^{\omega}(\vec{Q}, \Omega)\right]_{\alpha \beta \sigma \tau}=} \\
& \quad\left[\boldsymbol{\gamma}_{\vec{q} \vec{q}^{\prime \prime}}^{\omega}(\vec{Q}, \Omega)\right]_{\alpha \beta i j}\left[\boldsymbol{\Gamma}_{\vec{q}^{\prime \prime} \vec{q}^{\prime}}^{\omega}(\vec{Q}, \Omega)\right]_{i j \sigma \tau},
\end{aligned}
$$

where summation over repeated indices is implied, compare Fig 1 For tensor products of 2nd rank tensors in retarded and advanced space, $\boldsymbol{B}, \boldsymbol{C}$ and $\boldsymbol{E}, \boldsymbol{F}$, respectively, we have the multiplication rule

$$
(\boldsymbol{B} \otimes \boldsymbol{E})(\boldsymbol{C} \otimes \boldsymbol{F})=(\boldsymbol{B C}) \otimes(\boldsymbol{E F}),
$$

where $\boldsymbol{B} \boldsymbol{C}$ and $\boldsymbol{E} \boldsymbol{F}$ denotes the standard (matrix) product between tensors of second rank. It follows the general identity

$$
\begin{gathered}
\boldsymbol{G}_{\vec{q}_{+}}^{\omega_{+}} \otimes\left(\boldsymbol{G}_{\vec{q}_{-}}^{\omega_{-}}\right)^{*}=\left(\boldsymbol{G}_{\vec{q}_{+}}^{\omega_{+}} \otimes \mathbb{1}-\mathbb{1} \otimes\left(\boldsymbol{G}_{\vec{q}_{-}}^{\omega_{-}}\right)^{*}\right) \\
\times\left(\mathbb{1} \otimes\left[\left(\boldsymbol{G}_{\vec{q}_{-}}^{\omega_{-}}\right)^{*}\right]^{-1}-\left[\boldsymbol{G}_{\vec{q}_{+}}^{\omega_{+}}\right]^{-1} \otimes \mathbb{1}\right)^{-1},
\end{gathered}
$$

which allows us, after integration over the momentum $\vec{q}^{\prime}$, to rewrite the Bethe-Salpeter equation (9) as a kinetic equation (a generalized Boltzmann equation) for the system's integrated intensity correlation tensor

$$
\boldsymbol{\Phi}_{\vec{q}}^{\omega}(\vec{Q}, \Omega)=\int \frac{d^{3} q^{\prime}}{(2 \pi)^{3}} \Gamma_{\vec{q} \vec{q}^{\prime}}^{\omega}(\vec{Q}, \Omega)
$$

Explicitly, the generalized Boltzmann equation reads,

$$
\begin{aligned}
& {\left[-\Delta g_{\omega} \mathbb{1} \otimes \mathbb{1}-\Delta \hat{\boldsymbol{L}}_{\vec{q}}+\Delta \boldsymbol{\Sigma}_{\vec{q}}^{\omega}\right] \boldsymbol{\Phi}_{\vec{q}}^{\omega}=} \\
& \Delta \boldsymbol{G}_{\vec{q}}^{\omega}\left[\mathbb{1} \otimes \mathbb{1}+\int \frac{\mathrm{d}^{3} q^{\prime \prime}}{(2 \pi)^{3}} \gamma_{\vec{q} \vec{q}^{\prime \prime}}^{\omega} \boldsymbol{\Phi}_{\vec{q}^{\prime \prime}}^{\omega}\right] .
\end{aligned}
$$

Here, we have introduced short-hand notations for certain differences and, for later use, sums of 4th rank tensors,

$$
\begin{aligned}
\Delta g_{\omega}(\Omega) & =g\left(\omega_{+}\right)-g^{*}\left(\omega_{-}\right) \\
\Delta \hat{\boldsymbol{L}}_{\vec{q}}(\vec{Q}) & =\hat{L}\left(\vec{q}_{+}\right) \otimes \mathbb{1}-\mathbb{1} \otimes\left(\hat{L}\left(\vec{q}_{-}\right)\right)^{*} \\
\Delta \boldsymbol{\Sigma}_{\vec{q}}^{\omega}(\vec{Q}, \Omega) & =\boldsymbol{\Sigma}_{\vec{q}_{+}}^{\omega_{+}} \otimes \mathbb{1}-\mathbb{1} \otimes\left(\Sigma_{\vec{q}_{-}}^{\omega_{-}}\right)^{*} \\
\Delta \boldsymbol{G}_{\vec{q}}^{\omega}(\vec{Q}, \Omega) & =\boldsymbol{G}_{\vec{q}_{+}}^{\omega_{+}} \otimes \mathbb{1}-\mathbb{1} \otimes\left(\boldsymbol{G}_{\vec{q}_{+}}^{\omega_{+}}\right)^{*} \\
\Pi \boldsymbol{\Sigma}_{\vec{q}}^{\omega}(\vec{Q} ; \Omega) & =\boldsymbol{\Sigma}_{\vec{q}_{+}}^{\omega_{+}} \otimes \mathbb{1}+\mathbb{1} \otimes\left(\Sigma_{\vec{q}_{-}}^{\omega}\right)^{*} \\
\Pi \boldsymbol{G}_{\vec{q}}^{\omega}(\vec{Q} ; \Omega) & =\boldsymbol{G}_{\vec{q}_{+}}^{\omega_{+}} \otimes \mathbb{1}+\mathbb{1} \otimes\left(\boldsymbol{G}_{\vec{q}_{-}}^{\omega}\right)^{*}
\end{aligned}
$$

which employ the definitions $g(\omega)=\left(\omega^{2} / c^{2}\right) \epsilon_{h}(\omega)$ and $\hat{L}(\vec{r})=\vec{\nabla} \times \vec{\nabla} \times$ with corresponding Fourier representation $\hat{L}(\vec{q})=-\vec{q} \times \vec{q} \times$.

The physical interpretation of the generalized Boltzmann equation, Eq. (14) starts with the long-time limit, i.e. small $\Omega$ expansion of the first term on the left-hand side (l.h.s.), $\Delta g_{\omega}(\Omega)=i g_{\omega}^{(0)}+g_{\omega}^{(1)} \Omega+\ldots$, where

$$
\begin{aligned}
& g_{\omega}^{(0)}=2 \frac{\omega^{2}}{c^{2}} \operatorname{Im}\left[\epsilon_{h}(\omega)\right] \\
& g_{\omega}^{(1)}=\operatorname{Re}\left[\partial_{\omega} \frac{\omega^{2}}{c^{2}} \epsilon_{h}(\omega)\right] .
\end{aligned}
$$

It is seen that the term linear in $\Omega, g_{\omega}^{(1)}$ corresponds to a rate of change of the correlation tensor $\boldsymbol{\Phi}_{\vec{q}}^{\omega}(\vec{Q}, \Omega)$ (term of $\mathrm{O}(\Omega)$ ), while the term of $O\left(\omega^{0}\right)$ describes absorption or emission by the host medium. The latter is non-vanishing only if $\operatorname{Im} \epsilon_{h}(\omega) \neq 0$. Similarly, the second term on the l.h.s. represents a drift term (first order in $\vec{q})$ for $\Phi_{\vec{q}}^{\omega}(\vec{Q}, \Omega)$. The third term on the l.h.s of Eq. (14), $\Delta \boldsymbol{\Sigma}_{\vec{q}}^{\omega}(\vec{Q}, \Omega)$, embodies single-particle scattering from the external random perturbation, while the terms on the right-hand side (r.h.s.) represent an effective two-particle collision integral induced by disorder averaging.

The physical interpretation of the generalized Boltzmann equation suggests the subsequent strategy for obtaining the solution in the hydrodynamic limit $(\Omega \rightarrow 0$, $\vec{Q} \rightarrow 0$ ), where the collective mode dynamics are governed by the conservation laws of the system. The electromagnetic wave equation (2) being 2 nd order in time implies (in a loss- and gainless medium) the local conservation of the energy density rather than the intensity ${ }^{8}$. Consequently, we will seek solutions for the energy density correlation tensor $\boldsymbol{P}$ and the energy current density correlation tensor $\boldsymbol{J}$, and recast the kinetic equation in terms of these quantities. This is possible because, as seen in section IV, $\boldsymbol{P}$ and $\boldsymbol{J}$ are the leading coefficients of the correlator $\boldsymbol{\Phi}_{\vec{q}}^{\omega}(\vec{Q}, \Omega)$ in a small $\vec{Q}, \Omega$ expansion. Before we develop this solution in Sections IV and V, we will derive in the next section an exact WI for vector waves, which relates the photon selfenergy $\boldsymbol{\Sigma}$ and the irreducible two-photon vertex $\boldsymbol{g} a m m a$ to each other. It embodies local energy conservation as well as disspation or gain induced deviations in a field theoretical way.

\section{WARD IDENTITY}

The derivation of the WI for disordered electronic systems has been demonstrated for the first time by Vollhard and Wölfle $e^{43,44}$ using a diagrammatic approach. A corresponding WI for scalar classical waves has been derived, using an algebraic approach, by Barabanenkov et

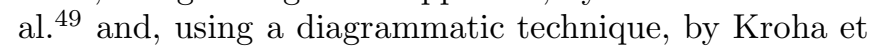

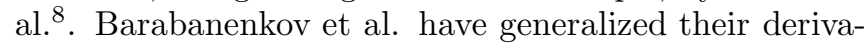
tion to electromagnetic waves 50.51 . Subsequently, the correct form of this WI has been the subject of heated debate ${ }^{52.53 .54}$, where a consensus has been reached in Ref. 54. However, to date, frequency dependent and/or complex dielectric functions have not been included in the derivation of WI for classical waves. Neither have all the 
implications of the WI on the renormalization of transport quantities been discussed.

Therefore, the aim of this section is to derive a WI for electromagnetic waves in the presence of frequency dependent, complex dielectric functions. In the following proof, we choose to follow the approach by Barabanenkov

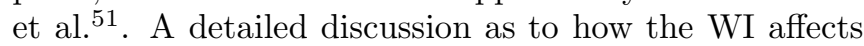
the various transport properties is postponed to sections IV and V.

We start from the Green's tensor before impurity averaging whose equation of motion (see Eqs. (2) and (3)) we write as

$$
\begin{aligned}
& \left(\hat{\boldsymbol{L}}\left(\vec{r}_{1}\right)+g\left(\omega_{1}\right)-h\left(\omega_{1}\right) V\left(\vec{r}_{1}\right)\right) \\
& \quad \times \tilde{\boldsymbol{G}}\left(\vec{r}_{1}, \vec{r}_{2}, \omega_{1}\right)=\delta\left(\vec{r}_{1}-\vec{r}_{2}\right) \mathbb{1} .
\end{aligned}
$$

Here, we have introduced the, in general, complex quantity

$$
h(\omega)=-\left(\epsilon_{s}(\omega)-\epsilon_{h}(\omega)\right)\left(\omega^{2} / c^{2}\right) .
$$

Multiplying Eq. 223) with $h\left(\omega_{2}\right)^{*} \tilde{\boldsymbol{G}}^{*}\left(\vec{r}_{3}, \vec{r}_{4}, \omega_{2}\right)$ within the appropriate tensor subspace yields

$$
\begin{gathered}
{\left[h^{*}\left(\omega_{2}\right) \hat{\boldsymbol{L}}\left(\vec{r}_{1}\right) \otimes \mathbb{1}+g\left(\omega_{1}\right) h^{*}\left(\omega_{2}\right) \mathbb{1} \otimes \mathbb{1}\right.} \\
\left.-h\left(\omega_{1}\right) h^{*}\left(\omega_{2}\right) V\left(\vec{r}_{1}\right) \mathbb{1} \otimes \mathbb{1}\right] \\
\times \tilde{\boldsymbol{G}}\left(\vec{r}_{1}, \vec{r}_{2} ; \omega_{1}\right) \otimes \tilde{\boldsymbol{G}}^{*}\left(\vec{r}_{3}, \vec{r}_{4} ; \omega_{2}\right) \\
=h^{*}\left(\omega_{2}\right) \delta\left(\vec{r}_{1}-\vec{r}_{2}\right) \mathbb{1} \otimes \tilde{\boldsymbol{G}}^{*}\left(\vec{r}_{3}, \vec{r}_{4} ; \omega_{2}\right) .
\end{gathered}
$$

In the time reversed case, i.e. starting with the complex conjugated equation, an analogous procedure leads to

$$
\begin{gathered}
{\left[h\left(\omega_{1}\right) \mathbb{1} \otimes \hat{\boldsymbol{L}}^{*}\left(\vec{r}_{3}\right)+g\left(\omega_{2}\right) h\left(\omega_{1}\right) \mathbb{1} \otimes \mathbb{1}\right.} \\
\left.-h\left(\omega_{1}\right) h^{*}\left(\omega_{2}\right) V\left(\vec{r}_{3}\right) \mathbb{1} \otimes \mathbb{1}\right] \\
\times \tilde{\boldsymbol{G}}\left(\vec{r}_{1}, \vec{r}_{2} ; \omega_{1}\right) \otimes \tilde{\boldsymbol{G}}^{*}\left(\vec{r}_{3}, \vec{r}_{4} ; \omega_{2}\right) \\
=h\left(\omega_{1}\right) \delta\left(\vec{r}_{3}-\vec{r}_{4}\right) \tilde{\boldsymbol{G}}\left(\vec{r}_{1}, \vec{r}_{2} ; \omega_{1}\right) \otimes \mathbb{1} .
\end{gathered}
$$

Upon substracting Eqs. (25) and Eq. (26), followed by averging over disorder and taking the limit of $\vec{r}_{1} \rightarrow \vec{r}_{3}$, we combine retarded and advanced quantities to what Barabenenkov et al $\stackrel{51}{w}$ refer to as the pre-Ward identity.

$$
\begin{aligned}
& 0=\lim _{\vec{r}_{1} \rightarrow \vec{r}_{3}}\left\{\left(\left[h^{*}\left(\omega_{-}\right) \hat{\boldsymbol{L}}\left(\vec{r}_{1}\right) \otimes \mathbb{1}-h\left(\omega_{+}\right) \mathbb{1} \otimes \hat{\boldsymbol{L}}^{*}\left(\vec{r}_{3}\right)\right]\right.\right. \\
& \left.+\left[g\left(\omega_{+}\right) h^{*}\left(\omega_{-}\right)-g^{*}\left(\omega_{-}\right) h\left(\omega_{+}\right)\right] \mathbb{1} \otimes \mathbb{1}\right) \\
& \left\langle\tilde{\boldsymbol{G}}\left(\vec{r}_{1}, \vec{r}_{2} ; \omega_{+}\right) \otimes \tilde{\boldsymbol{G}}^{*}\left(\vec{r}_{3}, \vec{r}_{4} ; \omega_{-}\right)\right\rangle \\
& -\left\langle h^{*}\left(\omega_{-}\right) \delta\left(\vec{r}_{1}-\vec{r}_{2}\right) \mathbb{1} \otimes \tilde{\boldsymbol{G}}^{*}\left(\vec{r}_{3}, \vec{r}_{4} ; \omega_{-}\right)\right. \\
& \left.\left.-h\left(\omega_{+}\right) \delta\left(\vec{r}_{3}-\vec{r}_{4}\right) \tilde{\boldsymbol{G}}\left(\vec{r}_{1}, \vec{r}_{2} ; \omega_{+}\right) \otimes \mathbb{1}\right\rangle\right\}
\end{aligned}
$$

The final step in obtaining the WI is to transfer the pre-Ward identity to momentum space and to cancel or simplify several terms with the help of both the BetheSalpeter equation and the generalized Boltzmann equation (for details we refer to the work of Barabanenkov et al $\sqrt[51]{1})$.

This finally yields the Ward identity for electromagnetic waves with complex frequency dependent dielectric functions,

$$
\begin{aligned}
& \Delta \boldsymbol{\Sigma}_{\vec{q}}^{\omega}(\vec{Q} ; \Omega)-\int \frac{\mathrm{d}^{3} q^{\prime}}{(2 \pi)^{3}} \Delta \boldsymbol{G}_{\vec{q}^{\prime}}^{\omega}(\vec{Q} ; \Omega) \boldsymbol{\gamma}_{\vec{q}^{\prime} \vec{q}}^{\omega}(\vec{Q}, \Omega) \\
& \quad=\frac{h\left(\omega_{+}\right)-h^{*}\left(\omega_{-}\right)}{h\left(\omega_{+}\right)+h^{*}\left(\omega_{-}\right)}\left[\Pi \boldsymbol{\Sigma}_{\vec{q}}^{\omega}(\vec{Q} ; \Omega)\right. \\
& \left.\quad+\int \frac{\mathrm{d}^{3} q^{\prime}}{(2 \pi)^{3}} \Pi \boldsymbol{G}_{\vec{q}^{\prime}}^{\omega}(\vec{Q} ; \Omega) \boldsymbol{\gamma}_{\vec{q}^{\prime} \vec{q}}^{\omega}(\vec{Q}, \Omega)\right]
\end{aligned}
$$

As compared to the case of electronic wave propagation in a disordered solid ${ }^{43.44}$, the non-zero r.h.s. of Eq. (28) constitutes a novel term which originates from the dependence of the "scattering potential" $\left(\omega^{2} / c^{2}\right)\left(\epsilon_{s}(\omega)-\right.$ $\left.\epsilon_{h}(\omega)\right) V(\vec{r})$ on the light frequency as well as from its possible imaginary part, and will lead below to a renormalization of the energy transport velocity ${ }^{5.7 .8}$. In fact, in the case of frequency independent and real dielectric constant, we have that the prefactor of this term, $\left(h\left(\omega_{+}\right)-h^{*}\left(\omega_{-}\right)\right) /\left(h\left(\omega_{+}\right)+h^{*}\left(\omega_{-}\right)\right)=4 \omega \Omega /\left(4 \omega^{2}+\Omega^{2}\right)$, takes on a form that has been discovered earlier ${ }^{8}$. Since in this case the r.h.s. of Eq. [28] is $\propto \Omega$ it leads to a renormalization of the energy transport velocity $\stackrel{8}{ }$. However, in the present case of absorptive or amplifying media, the r.h.s. contributes also in zero-th order in $O \Omega$, signaling that absorption and gain induce more severe effects than renormalizing the energy transport velocity, namely a mass term for the diffusion modes, see below.

\section{CONTINUITY EQUATION}

We now proceed with solving the kinetic equation (14). From the 2nd order wave equation (2) it follows that for a homogeneous system $\left(\epsilon(\vec{r})=\epsilon_{h}=\right.$ const. $)$ the quantities

$$
\begin{aligned}
& \rho_{E}=i \frac{\sqrt{\epsilon_{h}}}{c}\left(\vec{E}^{\dagger} \cdot \frac{\partial \vec{E}}{\partial t}-\frac{\partial \vec{E}^{\dagger}}{\partial t} \cdot \vec{E}\right) \\
& \vec{j}_{E}=i \frac{c}{\sqrt{\epsilon_{h}}}\left(\vec{E}^{\dagger} \cdot(\nabla \vec{E})-\left(\nabla \vec{E}^{\dagger}\right) \cdot \vec{E}\right)
\end{aligned}
$$

obey the continuity equation $\partial \rho_{E} / \partial t+\nabla \cdot \vec{j}_{E}=0$, and may be interpreted as energy density and energy current density, respectively, where $\bar{c}=c / \sqrt{\operatorname{Re} \epsilon_{h}(\omega)}$ is the phase velocity of the homogeneous medium. In order to obtain a similar relation for correlation functions in a random medium we, therefore, define the energy densityenergy density correlation tensor $\boldsymbol{P}_{\mathrm{E}}^{\omega}$, and the longitudinal energy current-energy density correlation tensor $\boldsymbol{J}_{\mathrm{E}}^{\omega}$ 
in Fourier representation as

$$
\begin{aligned}
& \boldsymbol{P}_{\mathrm{E}}^{\omega}(\vec{Q}, \Omega)=\omega^{2} \boldsymbol{c}_{\mathrm{P}}^{-2} \int \frac{d^{3} q}{(2 \pi)^{3}} \boldsymbol{\Phi}_{\vec{q}}^{\omega}(\vec{Q}, \Omega), \\
& \boldsymbol{J}_{\mathrm{E}}^{\omega}(\vec{Q}, \Omega)=\omega \boldsymbol{v}_{\mathrm{E}}(\omega) \boldsymbol{c}_{\mathrm{P}}^{-1} \int \frac{d^{3} q}{(2 \pi)^{3}}(\vec{q} \cdot \hat{Q}) \boldsymbol{\Phi}_{\vec{q}}^{\omega}(\vec{Q}, \Omega),
\end{aligned}
$$

where

$$
\boldsymbol{c}_{\mathrm{P}}=\bar{c}^{2}\left[\mathbb{1}-\operatorname{Re}\left(\boldsymbol{\Sigma}_{\vec{k}=\omega / c}^{\omega}\right) /\left(\omega^{2} / c^{2}\right)\right]^{-1}
$$

defines the averaged phase velocity tensor in the random medium ${ }^{6.8}$, and the energy transport velocity tensor $\boldsymbol{v}_{\mathrm{E}}(\omega)$ is to be identified below. We stress that in Eqs. (31) and (32) the products on the r.h.s. are contractions. For instance, in full index notation Eq. (31) reads

$$
\left[P_{\mathrm{E}}^{\omega}(\vec{Q}, \Omega)\right]_{\alpha \beta}=\omega^{2}\left[c_{\mathrm{P}}^{-2}\right]_{i j}\left[\int \frac{d^{3} q}{(2 \pi)^{3}} \boldsymbol{\Phi}_{\vec{q}}^{\omega}(\vec{Q}, \Omega)\right]_{i j \alpha \beta},
$$

where summation over repeated indices is implied. A corresponding expression can be written down for the current density tensor, Eq. (32).

The expressions Eqs. (31), (32) are the leading terms of a small $\Omega, \vec{Q}$ expansion of $\boldsymbol{\Phi}^{45.47}$, analogous to the so-called $\mathrm{P}_{1}$-approximation to the standard Boltzmann equation ${ }^{48}$,

$$
\begin{aligned}
\boldsymbol{\Phi}_{\vec{q}}^{\omega}(\vec{Q}, \Omega) \approx & \Delta \boldsymbol{G}_{\vec{q}}^{\omega}(0,0)\left[\tilde{P}_{E}^{\omega}(\vec{Q}, \Omega) \boldsymbol{A}_{0}^{-1}\right. \\
& \left.+(\vec{q} \cdot \vec{Q}) \tilde{J}_{E}^{\omega}(\vec{Q}, \Omega) \boldsymbol{A}_{1}^{-1}\right]+O\left(\Omega, \vec{Q}^{2}\right)(35) \\
\boldsymbol{A}_{0}= & \int d^{3} q \Delta \boldsymbol{G}_{\vec{q}}^{\omega}(0,0) \\
\boldsymbol{A}_{1}= & \int d^{3} q(\hat{q} \cdot \hat{Q})^{2} \Delta \boldsymbol{G}_{\vec{q}}^{\omega}(0,0)
\end{aligned}
$$

The tensor coefficients $\boldsymbol{A}_{0}$ and $\boldsymbol{A}_{1}$ above can be computed by projecting $\boldsymbol{\Phi}_{\vec{q}}^{\omega}(\vec{Q}, \Omega)$ onto its 0th and 1st moments with respect to the longitudinal current vertex $(\vec{q} \cdot \hat{Q})$, i.e. onto $\boldsymbol{P}_{\mathrm{E}}^{\omega}(\vec{Q}, \Omega)$ and $\boldsymbol{J}_{\mathrm{E}}^{\omega}(\vec{Q}, \Omega)$ respectively.

The continuity equation for $\boldsymbol{P}_{E}$ and $\boldsymbol{J}_{E}$, can be derived by integrating the generalized Boltzmann equation, Eq. (14), over the momentum $\vec{q}$ and subsequent application of the WI, Eq. (28). Upon considering the long-time, long-distance limit, we arrive at

$$
\begin{aligned}
& -\frac{1}{2} g_{\omega}^{(1)}[\mathbb{1} \otimes \mathbb{1}+\boldsymbol{\Delta}(\omega)] \Omega \boldsymbol{P}_{\mathrm{E}}^{\omega}+\omega\left[\boldsymbol{c}_{\mathrm{P}} \boldsymbol{v}_{\mathrm{E}}(\omega)\right]^{-1} Q \boldsymbol{J}_{\mathrm{E}}^{\omega}= \\
& \omega^{2} \boldsymbol{c}_{p}^{-2} \int d^{3} q \Delta \boldsymbol{G}_{\vec{q}}^{\omega}(\vec{Q}, \Omega)+\frac{1}{2} i\left[g_{\omega}^{(0)} \mathbb{1} \otimes \mathbb{1}+\boldsymbol{\Lambda}(\omega)\right] \boldsymbol{P}_{\mathrm{E}}^{\omega}
\end{aligned}
$$

The renormalization terms $\boldsymbol{\Delta}(\omega)$ and $\boldsymbol{\Lambda}(\omega)$ originate from the nonzero l.h.s. of the WI, Eq. (28), and are defined as

$$
\begin{aligned}
& \Delta(\omega)=\Delta^{(1)}(\omega)+\Delta^{(2)}(\omega) \\
& \boldsymbol{\Lambda}(\omega)=\frac{a_{\omega}^{(0)} g_{\omega}^{(1)}}{a_{\omega}^{(1)}} \Delta^{(1)}(\omega),
\end{aligned}
$$

with the explicit forms of $\Delta^{(1)}(\omega)$ and $\Delta^{(2)}(\omega)$ given by

$$
\begin{gathered}
\Delta^{(1)}(\omega)=-\frac{a_{\omega}^{(1)}}{g_{\omega}^{(1)}} \int \frac{d^{3} q}{(2 \pi)^{3}}\left\{\Pi \boldsymbol{\Sigma}_{\vec{q}}^{\omega}(0,0) \Delta \boldsymbol{G}_{\vec{q}}^{\omega}(0,0)\right. \\
\left.+\int \frac{\mathrm{d}^{3} q^{\prime}}{(2 \pi)^{3}} \Pi \boldsymbol{G}_{\vec{q}^{\prime}}^{\omega}(0,0) \boldsymbol{\gamma}_{\vec{q}^{\prime} \vec{q}}^{\omega}(0,0) \Delta \boldsymbol{G}_{\vec{q}}^{\omega}(0,0)\right\} \boldsymbol{A}_{0}^{-1} \\
\Delta^{(2)}(\omega)=-i \frac{\mathrm{a}_{\omega}^{(0)}}{g_{\omega}^{(1)}} \int \frac{\mathrm{d}^{3} q}{(2 \pi)^{3}}\left\{\left[\partial_{\Omega} \Sigma_{\vec{q}}^{\omega}(0,0)\right] \Delta \boldsymbol{G}_{\vec{q}}^{\omega}(0,0)\right. \\
+\int \frac{\mathrm{d}^{3} q^{\prime}}{(2 \pi)^{3}}\left[\partial_{\Omega} \boldsymbol{G}_{\vec{q}^{\prime}}^{\omega}(0,0)\right] \boldsymbol{\gamma}_{\vec{q}^{\prime} \vec{q}}^{\omega}(0,0) \Delta \boldsymbol{G}_{\vec{q}}^{\omega}(0,0) \\
\left.+\int \frac{\mathrm{d}^{3} q^{\prime}}{(2 \pi)^{3}} \Pi \boldsymbol{G}_{\vec{q}^{\prime}}^{\omega}(0,0)\left[\partial_{\Omega} \gamma_{\vec{q}^{\prime} \vec{q}}^{\omega}(0,0)\right] \Delta \boldsymbol{G}_{\vec{q}}^{\omega}(0,0)\right\} \boldsymbol{A}_{0}^{-1} .
\end{gathered}
$$

In Eqs. (3842) we have expanded the prefactor

$$
a_{\omega}(\Omega) \equiv \frac{h\left(\omega_{+}\right)-h^{*}\left(\omega_{-}\right)}{h\left(\omega_{+}\right)+h^{*}\left(\omega_{-}\right)}=i a_{\omega}^{(0)}+a_{\omega}^{(1)} \Omega+\cdots,
$$

to leading order in $\Omega$, where we introduced

$$
\begin{aligned}
a_{\omega}^{(0)} & =\frac{\operatorname{Im}[h(\omega)]}{\operatorname{Re}[h(\omega)]} \\
a_{\omega}^{(1)} & =\frac{\operatorname{Re}[h(\omega)] \operatorname{Re}\left[\partial_{\omega} h(\omega)\right]+\operatorname{Im}[h(\omega)] \operatorname{Im}\left[\partial_{\omega} h(\omega)\right]}{2 \operatorname{Re}[h(\omega)]} .
\end{aligned}
$$

Eq. (38) takes the form of a continuity equation by identifying the energy transport velocity $\boldsymbol{v}_{\mathrm{E}}(\omega)$ such that the prefactors of the two terms on the l.h.s. are equal, and by multiplying with the inverse of that prefactor. In fact, this procedure defines the energy transport velocity,

$$
\boldsymbol{v}_{\mathrm{E}}(\omega)=\frac{\omega}{g_{\omega}^{(1)}}\left[\boldsymbol{c}_{\mathrm{P}}\right]^{-1}[\mathbb{1} \otimes \mathbb{1}+\boldsymbol{\Delta}(\omega)]^{-1},
$$

where the r.h.s. implies a contraction as explained in Eq. (31). The continuity equation then reads,

$$
\begin{aligned}
& \Omega P_{\mathrm{E}}^{\omega}+Q \boldsymbol{J}_{\mathrm{E}}^{\omega}= \\
& -\frac{2}{g_{\omega}^{(1)}}[\mathbb{1} \otimes \mathbb{1}+\Delta(\omega)]^{-1} \omega^{2} \boldsymbol{c}_{p}^{-2} \int d^{3} q \Delta \boldsymbol{G}_{\vec{q}}^{\omega}(\vec{Q}, \Omega) \\
& +\frac{1}{g_{\omega}^{(1)}} i[\mathbb{1} \otimes \mathbb{1}+\boldsymbol{\Delta}(\omega)]^{-1}\left[g_{\omega}^{(0)} \mathbb{1} \otimes \mathbb{1}+\boldsymbol{\Lambda}(\omega)\right] \boldsymbol{P}_{\mathrm{E}}^{\omega} .
\end{aligned}
$$

We want to emphasize that in the long-time, longdistance limit, the continuity equation, Eq. (47) is exact. Also note that in the disordered medium transport occurs, with different velocities, both perpendicular and parallel to the polarization directions of the in- as well as of the outgoing wave field, since each scattering process alters the polarization. Accordingly, the averaged transport velocity $\boldsymbol{v}_{\mathrm{E}}(\omega)$ is a 4 th rank tensor, as evidenced by Eq. (46). Similarly, in a transport theory of a vector field the diffusion coefficient $\boldsymbol{D}(\omega)$, the transport mean free path $\boldsymbol{\ell}_{\mathrm{T}}(\omega)$, the absorption/gain length $\boldsymbol{x}_{a}(\omega)$, given 
below by Eqs. (55), (56), and (57), respectively, and other transport quantities are tensors as well.

The microscopic derivation of the energy transport velocity, Eq. (46), and the continuity equation, Eq. (47), exhibit a number of important physical aspects:

(1) The frequency dependent renormalization $\boldsymbol{\Delta}(\omega)$ of the energy transport velocity tensor consists of the "dwell time" renomalization $\boldsymbol{\Delta}^{(1)}(\omega)$ alluded to in the introduction which in the case of independent scatterers may be traced to their internal (Mie) resonances.

(2) However, in the presence of a mismatch in absorption or amplification ("impedance mismatch") between the scatterer and the host medium (non-vanishing $a_{\omega}^{(0)}$ ), Eq. (46) features additional renormalization of the transport velocity. To the best of our knowledge, this renormalization has not been discussed before. Numerical results for the transport velocity in absorptive or active media will be presented in Section VI.

(3) The r.h.s. of the continuity equation, Eq. (47) features as the first term the source contribution expected for correlation functions. We find that in the presence of either absorption or gain the second term on the r.h.s. does not vanish. Moreover, a "dwell time" renormalization $\boldsymbol{\Lambda}(\omega) \propto \boldsymbol{\Delta}^{(1)}(\omega)$ occurs in the case of unequal absorption or gain characteristics of host medium and scatterer (non-vanishing $\left.a_{\omega}^{(0)}\right)$. While such a renormalization of either absorption or gain could be expected on physical grounds, our calculation constitutes the first quantitative analysis of this effect. Both absorption/gain related renormalization terms may have profound influence on lasing action in random dielectric media and further explorations are necessary.

\section{FICK'S LAW AND DIFFUSION POLE}

In order to close the set of equations for $\boldsymbol{P}_{\mathrm{E}}$ and $\boldsymbol{J}_{\mathrm{E}}$, another relation besides the continuity equation has to derived from the kinetic equation, Eq. (14), which relates the current correlator $\boldsymbol{J}_{\mathrm{E}}$ to the gradient of the density correlator $\boldsymbol{P}_{\mathrm{E}}$, i.e. a version of Fick's law for the correlation tensors. This can be achieved by first multiplying Eq. (14) with $\vec{q} \cdot \hat{Q}$, subsequent integration over the momenta $\vec{q}$ and employing the WI, Eq. (28). The resulting relation should be evaluated to first order in momentum $Q$ at zero external frequency, $\Omega=0$ (see also the book by Case and Zweife ${ }^{48}$ for a detailed discussion of this socalled $\mathrm{P}_{1}$-approximation). This procedure leads to Fick's law,

$$
Q[\boldsymbol{A}+\boldsymbol{\kappa}(\omega)] \boldsymbol{P}_{\mathrm{E}}^{\omega}=g_{\omega}^{(1)} \boldsymbol{\ell}^{-1}[\mathbb{1} \otimes \mathbb{1}+\boldsymbol{\Delta}(\omega)] \boldsymbol{J}_{\mathrm{E}}^{\omega},
$$

where the renormalization terms $\boldsymbol{A}$ and $\boldsymbol{\kappa}$ are given by

$$
\begin{aligned}
\boldsymbol{A} & =\int \frac{\mathrm{d}^{3} q}{(2 \pi)^{3}}(\vec{q} \cdot \hat{Q})^{2} \Delta \boldsymbol{G}_{\vec{q}}^{\omega}(0,0) \boldsymbol{A}_{0}^{-1} \\
\boldsymbol{\kappa}(\omega) & =i a_{\omega}^{(0)} \int \frac{\mathrm{d}^{3} q}{(2 \pi)^{3}}(\vec{q} \cdot \hat{\vec{Q}}) \partial_{\vec{Q}}\left[\Pi \boldsymbol{\Sigma}_{\vec{q}}^{\omega}(0,0)\right. \\
& \left.+\int \frac{\mathrm{d}^{3} q^{\prime}}{(2 \pi)^{3}} \Pi \boldsymbol{G}_{\vec{q}^{\prime}}^{\omega}(0,0) \boldsymbol{\gamma}_{\vec{q}^{\prime} \vec{q}}(0,0)\right] \cdot \hat{\vec{Q}} \Delta \boldsymbol{G}_{\vec{q}}^{\omega}(0,0) \boldsymbol{a}_{0}^{-1}
\end{aligned}
$$

The tensor quantity $\boldsymbol{\ell}^{-1}(\omega)$ (of dimension inverse length)

$$
\boldsymbol{\ell}^{-1}(\omega)=i g_{\omega}^{(0)} \mathbb{1} \otimes \mathbb{1}+\Delta_{\omega}^{(3)}+\boldsymbol{\ell}_{0}^{-1}(\omega)
$$

consists of a "standard" term $\boldsymbol{\ell}_{0}^{-1}(\omega)$ that would also be there in electron transport theory,

$$
\begin{aligned}
\boldsymbol{\ell}_{0}^{-1}(\omega)=- & \int \frac{\mathrm{d}^{3} q}{(2 \pi)^{3}} \int \frac{\mathrm{d}^{3} q^{\prime}}{(2 \pi)^{3}}(\vec{q} \cdot \hat{\vec{Q}})^{2} \Delta \boldsymbol{G}_{\vec{q}^{\prime}}^{\omega}(0,0) \\
& \times \boldsymbol{\gamma}_{\vec{q}^{\prime} \vec{q}}(0,0) \Delta \boldsymbol{G}_{\vec{q}}^{\omega}(0,0) \boldsymbol{a}_{0}^{-1} \\
+ & \int \frac{\mathrm{d}^{3} q}{(2 \pi)^{3}} \int \frac{\mathrm{d}^{3} q^{\prime}}{(2 \pi)^{3}}(\vec{q} \cdot \hat{\vec{Q}})\left(\vec{q}^{\prime} \cdot \hat{\vec{Q}}\right) \Delta \boldsymbol{G}_{\vec{q}}^{\omega}(0,0) \\
& \times \boldsymbol{\gamma}_{\vec{q} \vec{q}^{\prime}}(0,0) \Delta \boldsymbol{G}_{\vec{q}^{\prime}}^{\omega}(0,0) \boldsymbol{a}_{1}^{-1},
\end{aligned}
$$

and two additional contributions $g_{\omega}^{(0)} \mathbb{1} \otimes \mathbb{1}$ and $\boldsymbol{\Delta}_{\omega}^{(3)}$ that are non-vanishing only in the presence of either absorption or gain,

$$
\begin{aligned}
\boldsymbol{\Delta}_{\omega}^{(3)} & =-i A_{\omega}^{(0)} \int \frac{\mathrm{d}^{3} q}{(2 \pi)^{3}}(\vec{q} \cdot \hat{\vec{Q}})^{2}\left[\boldsymbol{\Pi} \boldsymbol{\Sigma}_{\vec{q}}^{\omega}(0,0)\right. \\
& \left.+\int \frac{\mathrm{d}^{3} q^{\prime}}{(2 \pi)^{3}} \boldsymbol{\Pi} \boldsymbol{G}_{\vec{q}^{\prime}}^{\omega}(0,0) \boldsymbol{\gamma}_{\vec{q}^{\prime} \vec{q}}(0,0)\right] \Delta \boldsymbol{G}_{\vec{q}}^{\omega}(0,0) \boldsymbol{A}_{1}^{-1} .
\end{aligned}
$$

Similar to the case of the energy transport velocity, any discrepancy between the absorption/gain characteristics of scatterers and host medium ("impedance mismatch" given by a non-vanishing $\left.a_{\omega}^{(0)}\right)$ leads to a renormalization of $\boldsymbol{\ell}^{-1}$. Although the renormalizations $\boldsymbol{\Delta}^{(3)}(\omega)$ and $\boldsymbol{\kappa}(\omega)$ originate from isotropic and anisotropic scattering effects, respectively, they contribute only in the presence of absorption/gain mismatch between scatterer and host medium. This is in contrast to earlier results by Livdan and Lisyansky ${ }^{55}$ for media with frequency independent absorbing spheres, due to the fact that these authors inconsistently employed the WI without absorption/gain when deriving the current-density relation Eq. (48).

Combining the continuity equation, Eq. (38), with the microscopic version of Fick's law, Eq. (48), we are finally in a position to solve for the energy density tensor in the long-time, long-distance limit

$$
\begin{gathered}
P_{\mathrm{E}}^{\omega}=\left(-\frac{i}{2} g_{\omega}^{(1)}(1+\Delta(\omega))\left(-i \Omega+\boldsymbol{D}\left(Q^{2}+\boldsymbol{x}_{a}^{-2}\right)\right)\right)^{-1} \\
\times \omega^{2} \boldsymbol{c}_{p}^{-2} \int d^{3} q \Delta \boldsymbol{G}_{\vec{q}}^{\omega}(0,0)
\end{gathered}
$$

which exhibits a familiar diffusion pole structure. In addition, the presence of absorption or gain leads to the 
appearance of a mass term $\boldsymbol{D}(\omega) / \boldsymbol{x}_{a}^{-2}(\omega)$. This term is absent in the work of Livdan and Lisyansky ${ }^{55}$, although they considered absorbing spheres, albeit within a scalar model. From Eq. (54) the diffusion tensor $\boldsymbol{D}(\omega)$ is explicitly given by

$$
\begin{aligned}
\boldsymbol{D}(\omega) & =\frac{2 i}{g_{\omega}^{(1)}}(\mathbb{1} \otimes \mathbb{1}+\Delta(\omega))^{-1} \boldsymbol{\ell}(\boldsymbol{A}+\boldsymbol{\kappa}(\omega)) \\
& \equiv \frac{1}{3} \boldsymbol{v}_{\mathrm{E}}(\omega) \boldsymbol{\ell}_{\mathrm{T}}(\omega),
\end{aligned}
$$

which establishes the transport mean free path tensor $\boldsymbol{\ell}_{\mathrm{T}}(\omega)$ as

$$
\boldsymbol{\ell}_{\mathrm{T}}(\omega)=3 \frac{2 i}{\omega} \boldsymbol{c}_{\mathrm{P}} \boldsymbol{\ell}(\boldsymbol{A}+\boldsymbol{\kappa}(\omega))
$$

Finally, the absorption/gain length tensor $x_{a}(\omega)$ explicitly reads

$$
\boldsymbol{x}_{a}^{2}=\frac{1}{3} \boldsymbol{\ell}_{A} \boldsymbol{\ell}_{T}
$$

where the absorption mean free path tensor $\boldsymbol{\ell}_{A}$ is given by

$$
\boldsymbol{\ell}_{A}=\frac{\omega}{g_{\omega}^{(0)}}(\mathbb{1} \otimes \mathbb{1}+\boldsymbol{\Lambda}(\omega))^{-1} .
$$

Eqs. (46), (55), (56), and (57) represent the central results of our investigation and make explicit how the various renormalizations discussed above affect the transport quantities of a disordered dielectric medium in the presence of linear absorption or gain. In the following section, we provide illustrations of these results for a system of dilute scatterers.

\section{NUMERICAL RESULTS}

As alluded to above, any numerical evaluation of Eqs. (46), (55), (56), and (57) requires the computation of consistent values for the self-energy tensor $\Sigma_{\vec{q}_{ \pm}}^{\omega_{ \pm}}$and the irreducible vertex tensor $\gamma_{\vec{q} \vec{q}^{\prime}}^{\omega}(\vec{Q}, \Omega)$. For dense systems and in the diffusive regime, a Coherent Potential Approximation has to be employed for both, the self-energy tensor and the irreducible vertex tensor. To date, such an approach has been carried out for scalar waves and in the presence of point scatterers only 8.46 . Moreover, near the Anderson localization transition, the irreducible vertex tensor is expected to exhibit critical behavior and more sophisticated schemes such as a self-consistent theory of localization have to be employed. Again, to date such a program has been realized for scaler waves and point scatterers only 8.43 .44 .

However, in realistic strongly scattering electromagnetic systems, the scatterers cannot be approximated by point scatterers. Instead, they exhibit internal (Mie) resonances which - besides the vectorial nature of the electromagnetic radiation itself - greatly complicate the approach discussed above. Therefore, we have chosen to

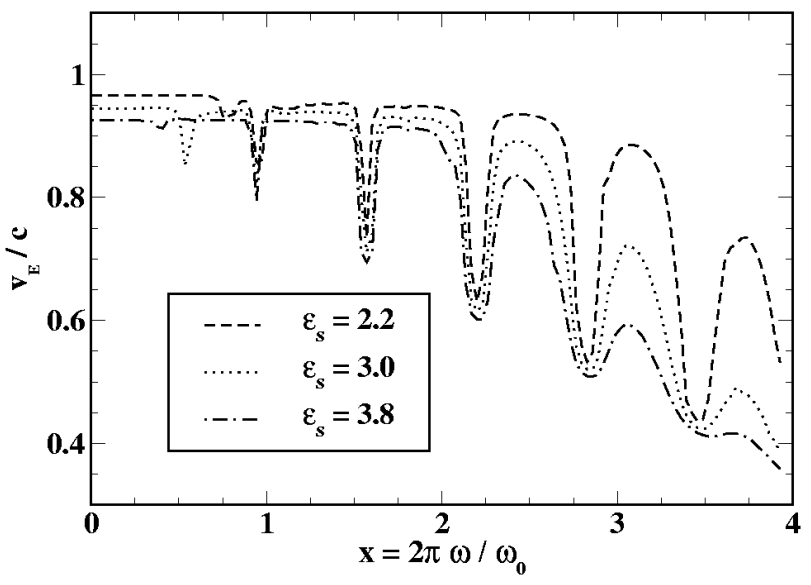

FIG. 2: Frequency dependence of the energy transport velocity $v_{\mathrm{E}}$ units of the vacuum speed of light for a dilute $(n=6 \%$ by volume) collection of spherical scatterers in air $\left(\epsilon_{h}=1\right)$ with different dielectric constants $\epsilon_{s}$ (as indicated). The frequency unit is $\omega_{0}=2 \pi c / r_{0}$ where $c$ is the vacuum speed of light and $r_{0}$ the radius of the scatterers. The pronounced dips correspond to the Mie resonances of the individual sphere and represent the "dwell time" effect discussed in the text.

illustrate our results in a simpler system consisting of a dilute collection of identical absorbing or amplifying spherical scatterers in a host medium without absorption or gain. In this case, the independent scatterer approximation applies and the self-energy as well as the irreducible vertex tensor may be expressed through the density $n$ of scatterers and the full t-matrix of such a scatterer according to 7.46

$$
\begin{aligned}
\Sigma_{\vec{q}_{ \pm}}^{\omega_{ \pm}} & =n \boldsymbol{t}_{\vec{q}_{ \pm}, \vec{q}_{ \pm}}\left(\omega_{ \pm}\right) \\
\boldsymbol{\gamma}_{\vec{q}, \vec{q}^{\prime}}(\vec{Q} ; \Omega) & =n \boldsymbol{t}_{\vec{q}_{+}, \vec{q}_{+}^{\prime}}\left(\omega_{+}\right) \otimes \boldsymbol{t}_{\vec{q}_{-}^{\prime}, \vec{q}_{-}}\left(\omega_{-}\right) .
\end{aligned}
$$

Explicit expressions for the t-matrix of a spherical scatterers are fairly involved and can be found in a recent work by K. Arya et al. ${ }^{56}$. Consequently, even within this approximation, all transport quantities have to be evaluated numerically.

Note that the integrations with respect to momentum of, e.g., certain convolutions of the irreducible Vertex $\boldsymbol{\gamma}$, which occur in the evaluation of the transport quantities, are in general ultraviolet divergent. These divergencies are remedied by applying the regularization scheme familiar from quantum electrodynamics 57 , i.e. by subtracting appropriate mass terms from the integrands such that after subtraction, these terms, and sufficiently many of their derivatives, do not diverge, thus making the integrals convergent. Note that the corresponding regularization masses in general have to be determined numerically, since the prefactors of the ultraviolet divergencies are known numerically only.

In the following we restrict ourselves to a discussion of the numerical results for the energy transport velocity tensor. We would like to point out that for isotropic 
dielectric materials, the energy transport velocity tensor becomes an isotropic tensor, so that we can further restrict our discussion to a single valued, frequency dependent energy velocity.

In Fig. 2] we display the frequency dependence of the energy transport velocity for a typical concentration of 6 $\%$ by volume of absorption-free scatterers with different (real) dielectric constants in an air background. Clearly visible are the pronounced dips in the energy transport velocity near frequencies that correspond to the single scatterer Mie resonances. As the dielectric constant increases, the Mie scattering becomes stronger and leads to correspondingly stronger renormalization of the energy transport velocity through the "dwell time" effect mentioned above. These data are, therefore, consistent with earlier results for scalar waves 5.7 and vector waves 14,15 . This behavior of the energy transport velocity has to be contrasted with the corresponding frequency dependence of the phase velocity, Eq. (33) in Fig. 3] Near the Mie resonances, this phase velocity may exhibit values that significantly exceed the vacuum speed of light. Since the phase velocity in a random medium does not correspond to a physical quantity, this behavior is not in conflict with the laws of relativity.

Adding linear gain to the system, dramatically modifies the situation. In Fig. 4 we show the frequency dependence of the energy transport velocity for the weakest scattering system of Fig. 2 linear gain is added to the scatterer. Although the concentration is only $n=6 \%$ by volume, adding a relatively small negative imaginary part to the dielectric constant results in a significant narrowing of the energy transport velocity resonances. This

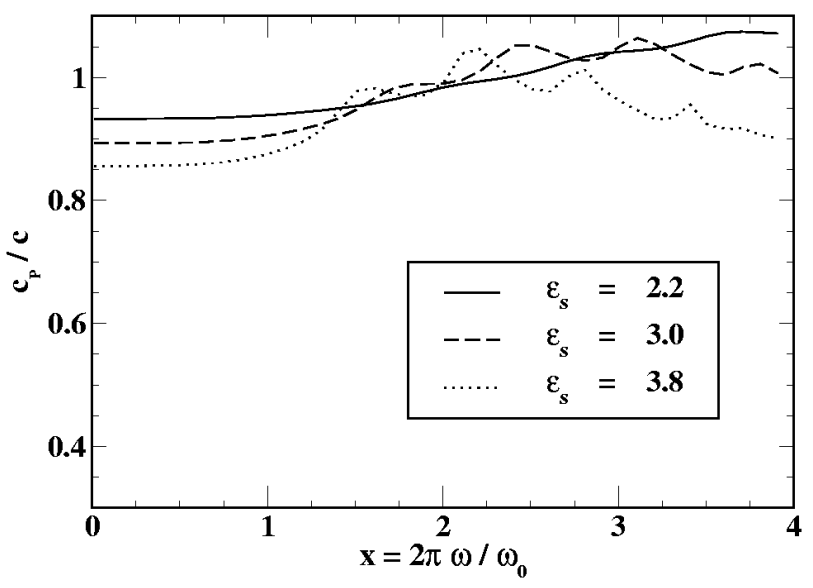

FIG. 3: Frequency dependence of the phase velocity $c_{\mathrm{P}}$ units of the vacuum speed of light for a dilute ( $n=6 \%$ by volume) collection of spherical scatterers in air $\left(\epsilon_{h}=1\right)$ with different dielectric constants $\epsilon_{s}$ (as indicated). The frequency unit is $\omega_{0}=2 \pi c / r_{0}$ where $c$ is the vacuum speed of light and $r_{0}$ the radius of the scatterers. Near Mie resonances, this velocity significantly exceeds the vacuum speed of light. The corresponding physical energy transport velocity is displayed in Fig. [2]

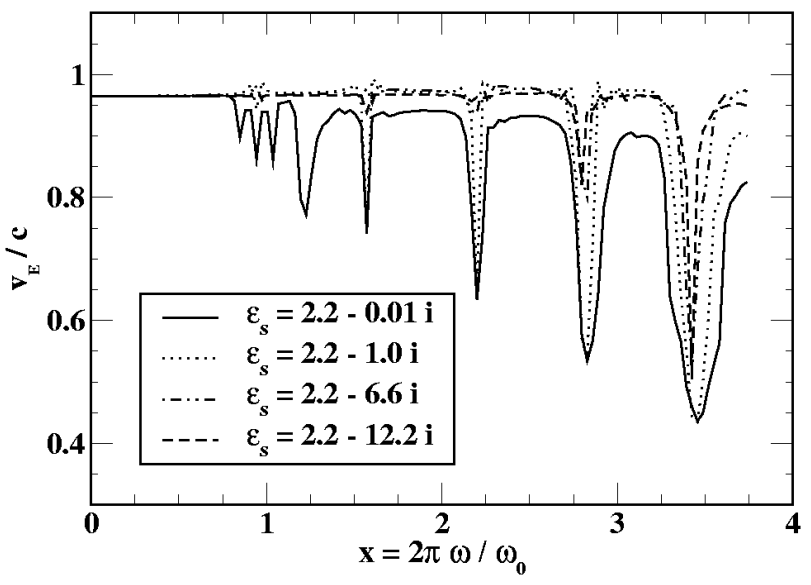

FIG. 4: Frequency dependence of the energy transport velocity $v_{\mathrm{E}}$ units of the vacuum speed of light for a dilute $(n=6 \%$ by volume) collection of spherical scatterers in air $\left(\epsilon_{h}=1\right)$ where the scatterer dielectric constant $\epsilon_{s}$ exhibits different amounts of linear gain (as indicated). The frequency unit is $\omega_{0}=2 \pi c / r_{0}$ where $c$ is the vacuum speed of light and $r_{0}$ the radius of the scatterers. For increasing gain, the dips in the energy transport velocity that are associated with the individual scatterers' Mie resonances exhibit considerable narrowing. This may be interpreted as a sign for the incipient onset of random lasing action in this system.

gain narrowing may be understood by noting that the Mie resonance dips in the transport velocity arise because of multiple reflection and interference of light within a single scatterer ("dwell time effect"). In the presence of gain, the relative importance of long wave paths for the interference processes is enhanced, leading to a narrowing of the resonance lines, analogous to the narrowing of the transmission lines in e Fabry-Perrot filter with increasing number of reflections. Increasing the gain to rather unrealistic values shows further narrowing of the resonances together with a slight increase in the energy transport velocity. It should be noted that the energy transport velocity retains physical values for all values of the gain that we have considered.

The opposite behavior occurs when we add linear absorption to the weakest scattering spheres of Fig. 2] This is illustrated in Fig. [5 where we display the frequency dependence of the energy transport for increasing values of linear absorption in this system. In this case, the dips in the energy transport velocity that are associated with the Mie resonances of the individual scatterers become washed out and disappear altogether for sufficiently large values of the linear absorption.

\section{CONCLUSION}

In conclusion, we have presented a microscopic approach to the calculation of transport quantities for electromagnetic waves propagating in disordered dielectric 


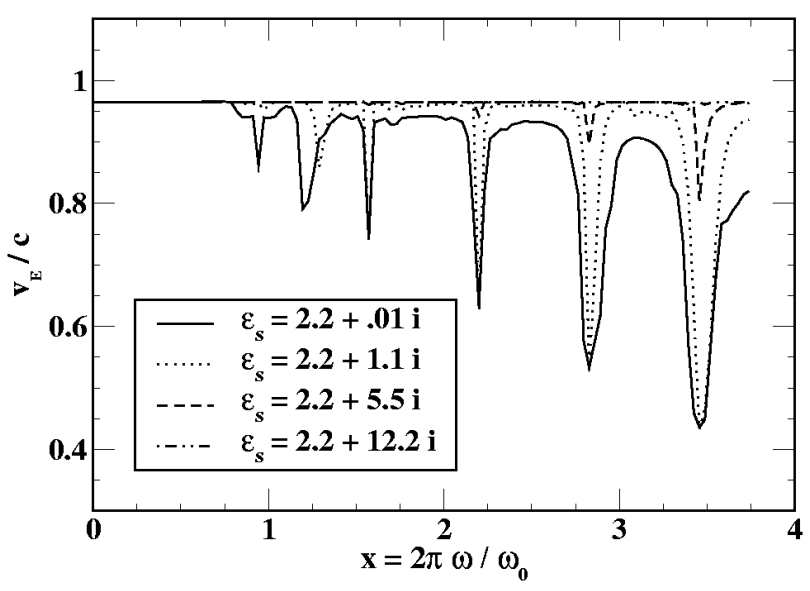

FIG. 5: Frequency dependence of the energy transport velocity $v_{\mathrm{E}}$ units of the vacuum speed of light for a dilute $(n=6 \%$ by volume) collection of spherical scatterers in air $\left(\epsilon_{h}=1\right)$ where the scatterer dielectric constant $\epsilon_{s}$ exhibits different amounts of linear absorption (as indicated). The frequency unit is $\omega_{0}=2 \pi c / r_{0}$ where $c$ is the vacuum speed of light and $r_{0}$ the radius of the scatterers. For increasing absorption, the dips in the energy transport velocity that are associated with the individual scatterers' Mie resonances become washed out and disappear altogether for sufficiently large absorption, as the wave propagation becomes overdamped and interference effects become impossible.

media which exhibit linear absorption or gain. The effects of energy conservation and of the violation of energy conservation in the presence of absorption or gain have been incorporated in the theory by means of an exact Ward identity. The kinetic equation for light in random media with absorption or gain has been derived and solved for the energy density correlation tensor, taking fully into account the vectorial nature of electromagnetic radiation. In this way we have, to the best of our knowledge, for the first time derived explicit expressions for the energy transport velocity tensor, the diffusion tensor, the mean free path tensor, and the absorption length tensor in absorbing/emitting media. All these quantities experience renormalization due to scattering or impedence mismatch between host medium and scatterer material.

Specifically, we have discussed the case of a dilute sys- tem of identical spherical scatterers within the framework of the independent scatterer approximation. These systems exhibit considerable dips in the energy transport velocity that can directly be traced to a well-known "dwell time" effect. However, adding linear gain to the scatterers dramatically modifies this situation and significant gain narrowing occurs already for relatively modest values of the scatterers concentration and the imaginary part of their dielectric constant. We interpret this behavior as due to an increase of the relative importance of wave paths with long path lengths in the medium, and a subsequent, gain-induced enhancement of interference effects, analogous to the Fabry-Perrot effect. The opposite effect occurs when absorption is added to scatterers and the resonances in the energy transport velocity are washed out and ultimately disappear altogether. In all cases, the energy transport velocity retains physical values, in contrast to the phase velocity.

As a systemiatic transport theory, expressing all physical quantities ultimately in terms of the single-photon selfenergy and the two-photon irreducible scattering vertex, our approach may be generalized to include interference effects like weak or strong localization, e.g. in the spirit of the self-consistent theory of Anderson localization that is well-known for electronic systems. Together with a replacement of the linear gain by a direct coupling of transport equations to the semiclassical laser rate equations this may provide a microscopic theory for the interplay of optical gain and Anderson localization of light. Similarly, the theory may be extended to include optically anisotropic materials such as scatterers immersed in a liquid crystal or Faraday-active scatterers.

\section{ACKNOWLEDGMENTS}

We thank C.M. Soukoulis for stimulating discussions. This project was supported in part by Deutsche Forschungsgemeinschaft (DFG) through Research Unit 557 grant KR 1726/2 (A.L., J.K.) and by grant KR $1726 / 3$ (J.K.) as well as by the Emmy-Noether program of the DFG through grant Bu 1107/2-3 (K.B.).
1 Y. Kuga and J. Ishimaru, J. Opt. Soc. Am. B 1, 831 (1984)

2 M.P. van Albada and A. Lagendijk, Phys. Rev. Lett. 55, 2692 (1985)

3 P.E. Wolf and G. Maret, Phys. Rev. Lett. 55, 2696 (1985)

4 P.W. Anderson, Phys. Rev. 109, 1492 (1958)

5 M.P. van Albada, B.A. van Tiggelen, A. Lagendijk, and A. Tip, Phys. Rev. Lett. 66, 3122 (1991)

${ }^{6}$ B.A. van Tiggelen, A. Lagendijk, M.P. van Albada, and A. Tip, Phys. Rev. B 45, 12233 (1992)

7 B.A. van Tiggelen, A. Lagendijk, and A. Tip, Phys. Rev.
Lett. 71, 1284 (1993)

8 J. Kroha, C. M. Soukoulis, and P. Wölfle, Phys. Rev. B 47, 11093 (1993)

9 E. Abrahams, P.W. Anderson, D.C. Licciardello, and T.V. Ramakrishnan, Phys. Rev. Lett. 42, 673 (1979)

10 D.S. Wiersma, P. Bartolini, A. Lagendijk, and R. Righini, Nature 390, 671 (1997)

11 F. Scheffold, R. Lenke, R. Tweer, and G. Maret, Nature 398, 206 (1999)

12 D.S. Wiersma, J. Gomez Rivas, P. Bartolini, A. Lagendijk, 
and R. Righini, Nature 398, 207 (1999)

13 J. Gomez Rivas, R. Sprik, C.M. Soukoulis, K. Busch, and A. Lagendijk, Europhys. Lett. 48, 22 (1999)

${ }^{14}$ K. Busch and C.M. Soukoulis, Phys. Rev. Lett. 75, 3442 (1995)

15 K. Busch and C.M. Soukoulis, Phys. Rev. B 54, 893 (1996)

16 F. Erbacher, R. Lenke, and G. Maret, Europhys. Lett. 21, 551 (1993)

17 R. Lenke, R. Lehner, and G. Maret, Europhys. Lett. 52, 620 (2000)

18 G. Labeyrie, C. Miniatura, C.A. Müller, O. Sigwarth, D. Delande, and R. Kaiser, Phys. Rev. Lett. 89, 163901 (2002)

19 G. Rikken and B.A. van Tiggelen, Nature 381, 54 (1996)

20 R. Sapienza, S. Mujumdar, C. Cheung, A.G. Yodh, and D.S. Wiersma, Phys. Rev. Lett. 92, 033903 (2004)

${ }^{21}$ B.A. van Tiggelen, R. Maynard, and A. Heiderich, Phys. Rev. Lett. 77, 639 (1996)

${ }^{22}$ H. Stark and T.C. Lubensky, Phys. Rev. Lett. 77, 2229 (1996)

23 K. Busch and S. John, Phys. Rev. Lett. 83, 967 (1999)

24 V.S. Letokhov, Sov. Phys. JETP 26, 835 (1968)

25 N.M. Lawandy, R.M. Balachandran, A.S.L. Gomes, and E. Sauvain, Nature 368, 436 (1994)

26 D.S. Wiersma and A. Lagendijk, Phys. Rev. Lett. 75, 1739 (1995)

27 S. Mujumdar, M. Ricci, R. Torre, and D.S. Wiersma, Phys. Rev. Lett. 93, 053903 (2004)

28 H. Cao, Y.G. Zhao, S.T. Ho, E.W. Seelig, Q.H. Wang, and R.P.H. Chang, Phys. Rev. Lett. 82, 2278 (1999)

29 H. Cao, J. Y. Xu, D. Z. Zhang, S. H. Chang, S. T. Ho, E. W. Seelig, X. Liu, R. P. H. Chang, Phys. Rev. Lett. 84, 5584 (2000).

30 S.V. Frolov, Z.V. Vardeny, K. Yoshino, A. Zakhidov, and R.H. Baughman, Phys.Rev. B 59, R5284 (1999)

31 H. Cao, Y. Ling, J. Y. Xu, and C. Q. Cao, Phys. Rev. Lett. 86, 4524 (2001).

${ }^{32}$ X. Jiang and C. M. Soukoulis Phys. Rev. Lett. 85, 70 (2000)

33 H. Cao, X. Jian, Y. Ling, J.Y. Xu, and C.M. Soukoulis, Phys. Rev. B 67 161101(R) (2003)
${ }^{34}$ H. Cao, Waves in Random Media 13, R1 (2003)

35 D. Wiersma, Nature 406, 132 (2000)

36 R.C. Polson and Z.V. Vardeny, Appl. Phys. Lett. 85, 1289 (2004)

37 B. Li et al., Opt. Lett. 27, 394 (2002)

38 S.M. Redmond et al., J. Opt. Soc. Am. B 21, 214 (2004)

39 D.S. Wiersma and A. Lagendijk, Phys. Rev. E 54, 4256 (1996)

40 L. Florescu and S. John, Phys. Rev. E 70, 036607 (2004)

41 L. Florescu and S. John, Phys. Rev. E 69, 046603 (2004)

42 D.S. Wiersma and S. Cavalieri, Nature 414, 708 (2001)

43 D. Vollhardt and P. Wölfle, Phys. Rev. Lett. 45, 842 (1980)

44 D. Vollhardt and P. Wölfle, Phys. Rev. B 22, 4666 (1980)

45 J. Kroha, Physica A 167, 231 (1990)

46 A. Gonis, Green functions for ordered and disordered systems, North-Holland (Amsterdam), 1992.

47 T. Kopp, J. Phys. C 17, 1897 (1984); 17, 1919 (1984).

48 K.M. Case and P.F. Zweifel, Linear Transport Theory, Addison-Wesley (Reading, Mass.) 1967

49 Zu.N. Barabanenkov and V.D. Ozrin, Phys. Lett. A 154, 38 (1991)

50 Yu.N. Barabanenkov and V.D. Ozrin, Phys. Lett. A 206, $116(1995)$

51 Yu.N. Barabanenkov, L.M. Zurk, and M.Yu. Barabanenkov, J. Electromagn. Waves Appl. 9, 1393 (1995)

52 H.T. Nieh, L. Chen, and P. Sheng, Phys. Rev. E 57, 1145 (1998)

53 Yu.N. Barabanenkov and V.D. Ozrin, Phys. Rev. E 64, 018601 (2001)

54 H.T. Nieh, L. Chen, and P. Sheng, Phys. Rev. E 64, 018602 (2001)

55 D. Livdan and A.A. Lisyansky, Phys. Rev. B 53, 14843 (1996)

56 K. Arya, Z. Su and J.L. Birman in World Scientific Series on Directions in Condensed Matter Physics - Vol. 8, World Scientific, 1990.

57 C. Itzikson and J.-B. Zuber, Quantum Field Theory (MacGraw-Hill, 1978). 